VIII ${ }^{\text {èmes }}$ Journées Nationales Génie Civil - Génie Côtier, Compiègne, 7-9 septembre 2004

\title{
Modélisation du transport de sédiments mixtes (vase et sable fin)
}

\author{
Benoît Waeles et Pierre Le Hir
}

\author{
IFREMER/DEL/EC/TP centre Ifremer de Brest BP70 29280 Plouzané
}

\begin{abstract}
Résumé
De nombreuses études de dynamique sédimentaire nécessitent la modélisation simultanée des transports de sable et de vase. Lorsque les sables sont fins, ils sont surtout transportés en suspension. Dans une première partie, il est montré qu'un modèle d'advection-diffusion de sable, assorti d'une loi d'érosion adaptée, reproduit les capacités de transport à l'équilibre données dans la littérature, à condition d'extrapoler convenablement la concentration. Puis est présentée la stratégie de modélisation du comportement des mélanges sable/vase, en distinguant un régime cohésif ou non cohésif, selon que la proportion de vase dépasse ou non une valeur critique. La méthode est appliquée à l'embouchure de la Seine, à l'aide du logiciel de calcul hydrosédimentaire SiAM-3D.
\end{abstract}

\begin{abstract}
$\underline{\text { Abstract }}$
Many problems of sediment dynamics require to account for transport of sand and mud, simultaneously. Fine sands are mainly transported in suspension. In a first part, it is shown that an advection-diffusion model can simulate classical equilibrium transport capacities, provided bottom concentration is appropriately extrapolated. Then the strategy for modelling the behaviour of sand/mud mixtures is presented, consisting of a distinction between cohesive or not cohesive regime, depending on the muddy fraction compared to a critical value. The method is applied to the mouth of the Seine estuary, by means of the SiAM-3D code.
\end{abstract}

\section{Introduction}

Pour décrire le comportement des sédiments, il est d'usage de distinguer les matériaux cohésifs (vases) des matériaux non cohésifs (sables ou éléments plus grossiers). Les vases se distinguent par leur capacité à floculer lorsqu'elles sont en suspension, et à se consolider lorsqu'elles sont déposées : leur érodabilité peut alors évoluer au cours du temps. En raison de la petite taille des particules élémentaires ou de la faible densité des flocs, le transport des sédiments cohésifs se fait essentiellement en suspension, leur vitesse de déplacement horizontal étant identique à celle de l'eau. Le comportement des sables est différent : pour un forçage donné les conditions de remaniement ne dépendent que des caractéristiques des grains, et la vitesse de chute de ces derniers est plus élevée, de telle sorte qu'ils se déplacent souvent par petits sauts ou par roulement sur le lit sédimentaire : ils ne connaissent pas la même vitesse moyenne que le fluide, et l'on ne sait pas décrire le détail de leurs mouvements.

Les stratégies de modélisation du transport de ces deux types de matériel particulaire sont très différentes. Ne pouvant décrire le mouvement des sables, on en quantifie directement le flux solide à l'aide d'une formulation semi-empirique de la capacité de transport de l'écoulement (ou flux solide à l'équilibre) que l'on relie à l'écoulement. Les évolutions des fonds (érosion/dépôt) sont alors déduites de la divergence des flux solides calculés, en respectant la conservation de la masse. Hors équilibre, on a introduit, mais seulement depuis les années 1980 le concept de "mise en régime" (e.g. Holly et Rahuel, 1990), qui permet de ne pas supposer qu'un déclenchement de transport de sable ne se fasse instantanément au taux observé à l'équilibre. Les vases, quant à elles, sont classiquement modélisées par résolution 
d'une équation d'advection/dispersion, dans laquelle on cherche à décrire l'évolution de la concentration en suspension, transportée à la vitesse du courant, sans supposer d'équilibre, et en se donnant un flux d'érosion par unité de surface de sédiment, paramétré en fonction de l'état de consolidation de celui-ci. Dans ce cas les évolutions des fonds se calculent par différence entre les flux d'érosion et de dépôt.

Si la différence d'approche se justifie par le mode de transport des différents matériaux, elle est d'abord "culturelle", la dynamique sédimentaire ayant d'abord été développée pour les déplacements de matériaux grossiers en milieu fluvial où l'écoulement est quasi stationnaire. S'agissant des sables fins, le mode de transport est majoritairement la suspension, la saltation n'intervenant que pour un nombre de Shields très proche de la valeur critique de mise en mouvement (les courbes de mise en mouvement et de mise en suspension convergent lorsque le nombre de Reynolds de la particule diminue (e.g. Van Rijn, 1989). Il est alors raisonnable de traiter le transport de ces sables fins comme celui des vases, i.e. par advection/dispersion, à condition de disposer d'une formulation adaptée pour les flux d'érosion ("pick up function") : c'est l'objet de la première partie de ce travail.

Mais surtout, dans de nombreuses configurations du milieu marin côtier, le fond est constitué de sédiments mixtes, soit sous forme de vrais mélanges de sables et de fines, soit sous forme de couches alternées plus ou moins fines de sable et de vase (exemples en France : Grande Vasière du golfe de Gascogne, baie du mont St-Michel, baie des Veys, embouchure de la Seine...). Il est donc crucial de pouvoir modéliser simultanément les deux types de sédiment. La présente étude vise à traiter le cas de mélanges entre sables fins et vases, en considérant que le sable est majoritairement transporté en suspension.

\section{$\underline{\text { 2.Modélisation du transport de sable fin en suspension }}$}

\subsection{Synthèse bibliographique : flux d'érosion versus concentration de référence}

Dans une première phase, nous avons exploré la possibilité de traiter la dynamique des sables fins en résolvant une équation d'advection-diffusion de la concentration en suspension. Si la résolution d'une telle équation est classique, avec quelques écueils (formulation de la diffusion turbulente, diffusion numérique des schémas d'advection...), l'application au transport de sédiment se heurte à la difficulté d'exprimer la condition à la limite du fond, c'est à dire à l'interface eau/sédiment. Deux tendances se dégagent :

-l'imposition d'une concentration "de référence" $\mathrm{C}_{\mathrm{a}}$ à une hauteur de référence $a$ au-dessus du fond, qui résulte de l'hypothèse que rapidement un équilibre se met en place entre dépôt près du fond et érosion. Cette concentration de référence est a priori exprimée en fonction des contraintes sur le fond $\tau$ et des caractéristiques du sédiment (e.g. Smith et Mac Lean, 1977). Lorsque l'équilibre se généralise à l'ensemble de la colonne d'eau (chute des particules compensée par le mélange vertical turbulent), l'équation de conservation de la concentration en suspension a une solution analytique proposée en 1938 par Rouse.

-une condition de flux à l'interface, résultant 1) d'un flux d'érosion empirique $\mathrm{E}$ qui dépend comme $\mathrm{C}_{\mathrm{a}}$ du forçage et des caractéristiques du sédiment et 2) d'un flux de dépôt déterminé comme le produit de la concentration près du fond par la vitesse de chute $\mathrm{W}_{\mathrm{s}}$. A l'équilibre, les échanges eau/sédiment se compensent de telle sorte que le flux net est nul, et l'on se ramène au cas précédent, avec $E=-W_{\mathrm{s}} \cdot \mathrm{C}_{\mathrm{a}}$. Hors équilibre, les flux ne se compensent pas nécessairement, et l'on peut avoir érosion ou dépôt résiduels. Cette approche est donc moins réductrice, et permet non seulement d'évaluer la concentration en suspension, mais aussi les érosions et les dépôts. 
Quelques (rares) fonctions d'érosion des sables (ou "pick-up functions") ont été proposées dans la littérature. Citons celle Chapalain et Thais (2000) inspirée de l'expression de la concentration de référence de Smith et Mac Lean (1977) :

$$
E=(1-p)\left(\frac{\gamma_{0} T}{1+\gamma_{0} T}\right) \cdot W_{s}
$$

avec : p porosité du sédiment

$\gamma_{0}$ paramètre empirique

T excès de cisaillement $=\left(\tau-\tau_{\mathrm{cr}}\right) / \tau_{\mathrm{cr}}$

$\tau_{\mathrm{cr}}$ cisaillement critique (selon relation de Shields)

ou encore celle de Nelson, reprise par Beach et Sternberg (1988): $E=(1-p)\left(\gamma_{1} T\right) \cdot W_{s}$.

On peut noter que pour les grandes valeurs de cisaillement, l'expression de Smith et Mac Lean présente une valeur plafond : Ws(1-p). On pourrait aussi mentionner les formulations de Van Rijn (1984) ou de Fredsoe et al. (1986).

Pour des écoulements non permanents (vagues symétriques ou asymétriques), Davies et Li (1997) ont comparé les résultats d'un modèle 1DV obtenus avec une approche concentration de référence, ou une approche flux d'érosion, sans conclure à une nette préférence entre les deux. Certains auteurs suggèrent d'ajouter au transport en suspension une contribution du charriage, représentant par exemple le transport entre le fond et la hauteur de référence a. Cette contribution est souvent considérée négligeable pour des sables fins. Davies et Li (1997) montrent dans le cas de mouvements oscillants que l'ajout d'un transport par charriage n'améliore pas les résultats.

\subsection{Validation 1DV de la modélisation du transport en suspension de sable par advection/diffusion, sous l'action d'un courant permanent}

Nous avons comparé les flux horizontaux de transport de sable déduits d'une modélisation de type "advection/diffusion" (modèle SiAM-1DV, Le Hir et al., 2001a) à des formules empiriques classiques pour les sédiments fins non cohésifs. L'exercice nous a montré une forte sensibilité des résultats au calcul du flux de dépôt près du fond : en effet, lorsque le régime permanent est atteint, le flux de dépôt doit égaler le flux d'érosion, fixé . Dans une colonne d'eau "discrétisée", ce flux de dépôt ne doit pas être exprimé en fonction d'une concentration au milieu de la maille inférieure, mais plutôt à la base de cette maille, sachant que la concentration augmente à l'infini (selon Rouse) en s'approchant du fond... Par ailleurs, il est essentiel que les transports calculés soient indépendants de la résolution verticale du modèle, celle-ci pouvant varier dans une simulation 3D. Nous avons supposé qu'au sein de la maille du fond le profil de type "Rouse" était valide, et en avons déduit une extrapolation de la concentration à une hauteur de référence à partir de la concentration dans la maille inférieure. Le flux de dépôt est exprimé à cette hauteur de référence qui constitue un paramètre du modèle (choisi inférieur à la demi-hauteur de la maille de fond). 


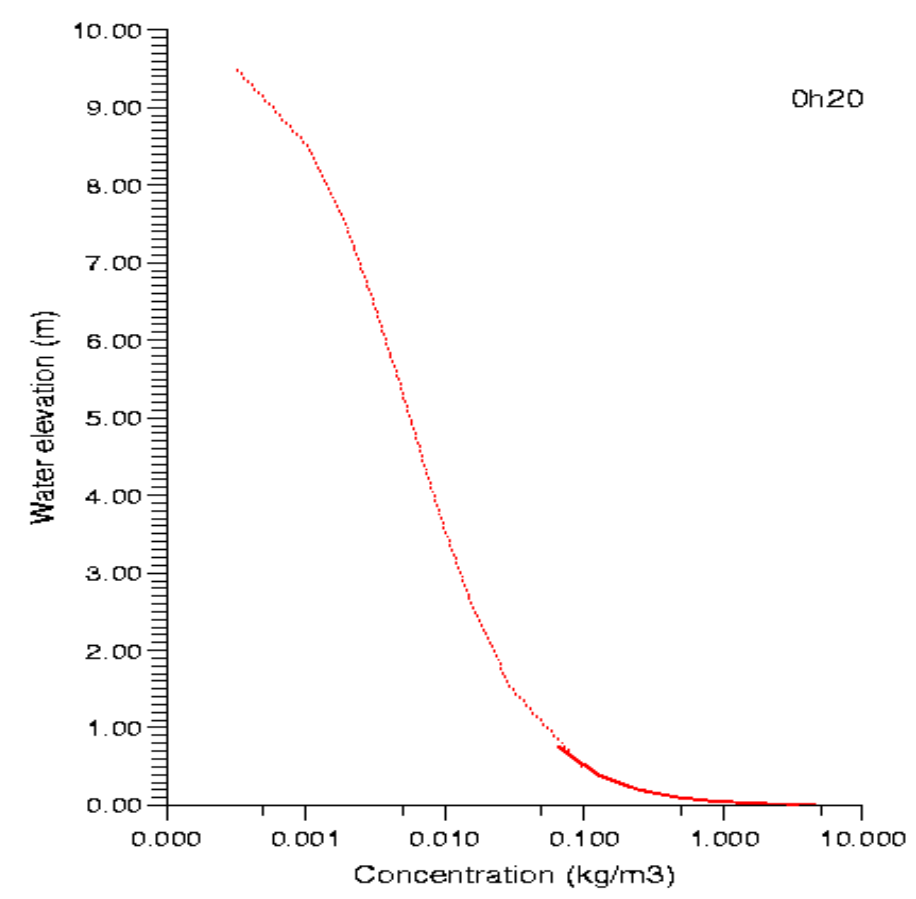

Figure 1 : exemple de profil à l'équilibre de concentration de sable en suspension, calculé avec le modèle Siam-1DV.

Courant moyen : $1,5 \mathrm{~m} . \mathrm{s}^{-1}$; hauteur d'eau : $10 \mathrm{~m}$; diamètre du sable : $200 \mu \mathrm{m}$;

Discrétisation verticale : $1 \mathrm{~m}$

Hauteur de référence (flux de dépôt) : $1 \mathrm{~cm}$

Le profil en gras dans la couche de fond sur la figure 1 est la solution analytique de Rouse : quelle que soit la résolution verticale du modèle, nous avons noté une bonne continuité entre le profil simulé numériquement et le profil de Rouse près du fond, et donc une indépendance du profil simulé vis-à-vis de la discrétisation.

Cependant le flux horizontal de sable, calculé directement comme $\sum_{k \text { couches }} u_{k} C_{k}$ s'est avéré très sensible à la discrétisation verticale : le problème a été résolu en calculant le flux dans la maille de fond à partir de l'intégrale $\int_{\text {ep.couche.fond }} u(z) c(z) d z$, en supposant $\mathrm{u}(\mathrm{z})$ logarithmique. Le calcul numérique de cette intégrale est réalisé en discrétisant la couche de fond selon un raffinement géométrique afin de mieux décrire les gradients croissants de la concentration vers le bas. Le flux résultant se stabilise alors rapidement.

La figure 2 permet de situer les flux de sable ainsi simulés, en choisissant l'expression de Beach et Sternberg (1988) pour le flux d'érosion (avec $\gamma_{1}=2,2.10^{-3}, \mathrm{p}=0,35$, a $=5 \mathrm{~mm}, \mathrm{~W}_{\mathrm{s}}$ selon Soulsby (1997) et $\tau_{\mathrm{cr}}$ selon Shields) parmi les formulations empiriques de capacités de transport total proposées par Engelund et Hansen (in Soulsby, 1997) ou par Van Rijn (ibidem), couramment utilisées pour des sables fins. On note qu'après raffinement de l'intégration dans la couche de fond le modèle est peu sensible à la discrétisation verticale. Le modèle de transport en suspension, formulé en advection/diffusion avec loi d'érosion, s'avère ainsi capable de reproduire les variations de transport solide telles qu'évaluées à partir des formules classiques qui ont été validées par des tests en laboratoire, dans la gamme d'incertitude qui les caractérise. 


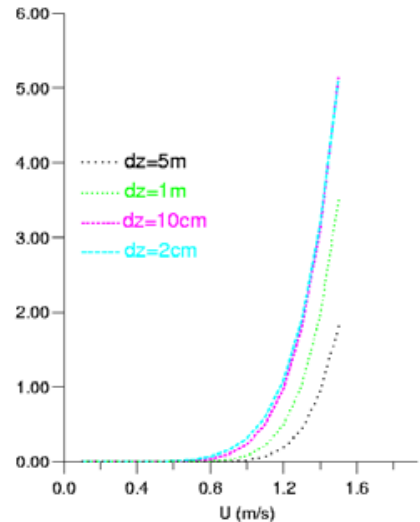

a) effet de la discrétisation verticale : le calcul du flux horizontal pour toute la colonne d'eau est $\sum_{\text {kcouches }} u_{k} C_{k}$

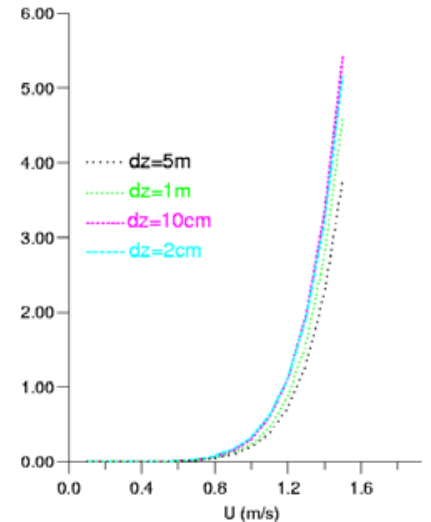

b) effet de la discrétisation verticale : le calcul du flux horizontal dans la couche de fond est une intégration en supposant un profil de Rouse

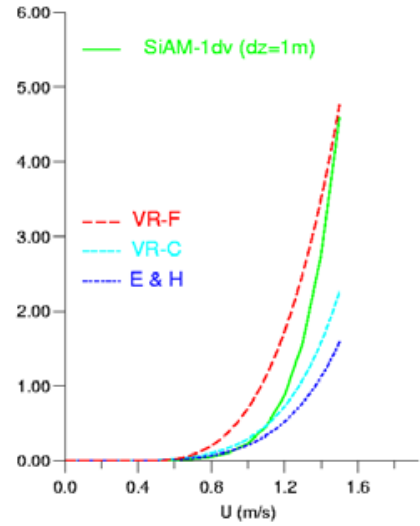

c)comparaison avec formules de transport classiques: -VR-F: Van Rijn 1984 simplifié

-VR-C: Van Rijn 1984 complet -E\&H: Engelund et Hansen 1972

Figure 2 :Simulation du flux de transport $\left(\mathrm{kg} \cdot \mathrm{m}^{-1} \cdot \mathrm{s}^{-1}\right)$ de sable $(\Phi=200 \mu \mathrm{m})$ en fonc-tion du courant, par intégration d'un modèle $1 D \mathrm{~V}$ (modèle SiAM-1DV).

\section{Comportement des sédiments mixtes sable/vase}

Les tests d'érodabilité de mélanges sable/vase sont peu nombreux. Pour un mélange plutôt sableux,la contrainte critique d'érosion augmente avec la fraction de particules fines (Mitchener et Torfs 1996, Panagiotopoulos et al. 1997). A mesure que la proportion de vase augmente, les fines remplissent les espaces entre les points de contact des grains de sable, réduisant la possibilité pour les grains de pivoter les uns par rapport aux autres: la mise en mouvement est alors plus difficile. A partir d'un certain pourcentage de vase, les grains de sable ne sont plus en contact les uns avec les autres et sont intégrés dans une matrice vaseuse et le comportement de l'ensemble devient cohésif. Selon Van Ledden et al. (2004) c'est le pourcentage d'argile granulométrique (fraction $<4 \mu \mathrm{m}$ ) qui serait le paramètre pertinent pour caractériser l'érodabilité d'un fond sédimentaire mixte. Toutefois, il est acceptable d'utiliser le pourcentage de vase $(\%<63 \mu \mathrm{m})$ si l'on considère que le rapport silt/argile varie peu pour des vases naturelles.

L'ajout de sable à un fond vaseux a également pour effet d'augmenter la tension critique d'érosion, mais de façon moins sensible (Mitchener et Torfs, 1996). Cette augmentation est attribuée à l'augmentation de densité de la mixture. La contrainte critique d'érosion serait maximale lorsque la proportion de fines est d'environ 40-50 \% (Mitchener et Torfs, 1996). Les mesures effectuées pour cette gamme de pourcentages sont cependant quasiment inexistantes.

Les processus de dépôt et de sédimentation constituant des fonds mixtes sable/vase sont mal connus. Durant la chute d'une mixture des grains de sable peuvent entrer en collision avec des flocs de vase et les casser, entraînant la formation de flocs plus petits et plus denses (Torfs et al., 1996). C'est le cas en particulier près du fond où s'effectue préférentiellement le transport du sable. Les tests en colonne de sédimentation font apparaître une ségrégation sable/vase dans le sédiment. Les grains de sable ont tendance à traverser la couche de vase fraîchement déposée et non encore consolidée. La présence de sable dans un fond vaseux tend à rendre plus dense ce fond et à accélérer sa consolidation.(Mitchener et Torfs, 1996): 
Le passage des grains de sable à travers la vase qui n'est pas encore structurée laisse des espaces facilitant le drainage, ce qui a tendance à compacter celle-ci.

\section{Modélisation du transport simultané sable/vase}

Chesher et Ockenden (1997) ont simulé le transport de sable et de vase dans le cas d'un simple canal, pour un courant uniforme, puis dans un cas réel, celui de l'estuaire de la Mersey (UK) . Un résultat important est que la non prise en compte des interactions sable/vase entraîne une large surestimation de l'érosion (et donc du transport), à la fois pour le sable et pour la vase. Van Ledden (2003) a également simulé conjointement les transports de sable et de particules fines en tenant compte de leurs interactions. En particulier il distingue deux régimes d'érosion : un régime non-cohésif lorsque le fond sédimentaire est constitué de moins de $30 \%$ de vase, un régime cohésif sinon. L’intégration des mélanges sable/vase dans nos codes de calcul SiAM s'inspire de l'approche de Van Ledden (2003) concernant le processus d'érosion, et est brièvement décrite ci-après.

\section{Transport simultané de sable et de vase dans la colonne d'eau.}

Suivant Chesher et Ockenden (1997) qui se basent sur des mesures réalisées en laboratoire et sur le terrain, on suppose que les grains de sable et les particules fines sont transportées indépendamment dans la colonne d'eau. Une équation d'advection-dispersion est résolue pour chaque fraction, caractérisée par sa vitesse de chute. Cette dernière est susceptible de varier en raison des phénomènes de floculation et d'entravement.

\section{Dépôt à l'interface eau/sédiment}

Sable et vase se déposent indépendamment. Le dépôt de sable est estimé a partir d'une concentration extrapolée près du fond $D_{\text {sable }}=W_{s}$. $C_{\text {fond, }}$ tandis que le dépôt de vase est calculé a l'aide de la loi classique de Krone : $D_{\text {vase }}=W_{s}$. $C_{\text {vase }} \cdot\left(1-\tau / \tau_{d}\right)$ si $\tau<\tau_{d}$ pour laquelle $\tau_{d}$ est choisie en fonction des options de tassement du modèle. $\mathrm{C}_{\text {vase }}$ est la concentration de particules fines en suspension dans la couche de fond, $\tau$ la contrainte de cisaillement sur le fond et $\tau_{\mathrm{d}}$ la contrainte critique pour le dépôt. Contrairement au cas du dépôt de sable il n'est pas nécessaire de calculer une concentration près du fond car le gradient vertical y est généralement faible ( $\mathrm{W}_{\mathrm{s}}$ petit)

\section{Erosion à l'interface eau/sédiment}

Conformément aux expériences de Torfs (1995, in Van Ledden 2001) et de Panagiotopoulos et al. (1997), on distingue un régime cohésif et un régime non cohésif, en fonction de la fraction massique de vase $F v$ du sédiment superficiel:

-régime non-cohésif $\left(F v<F v_{c r}\right.$ ). L'érosion des deux fractions (sable et vase) suit une loi d'érosion adaptée aux sables, par exemple celle de Beach et Sternberg (1988), au prorata des fractions respectives dans le sédiment superficiel. La contrainte critique varie en fonction de ces fractions conformément aux observations de Mitchener et Torfs (1996) et de Panagiotopoulos et al. (1997), ce qu'illustre la figure 3.

-régime cohésif $\left(F v>F v_{c r}\right)$. Une loi classique relative aux sédiments cohésifs est alors appliquée (par exemple Partheniades), ici encore au prorata des différentes fractions. Dans ce cas la contrainte critique peut varier en fonction de ces fractions, mais aussi de l'état de consolidation du sédiment (zone en grisé sur la figure 3). 


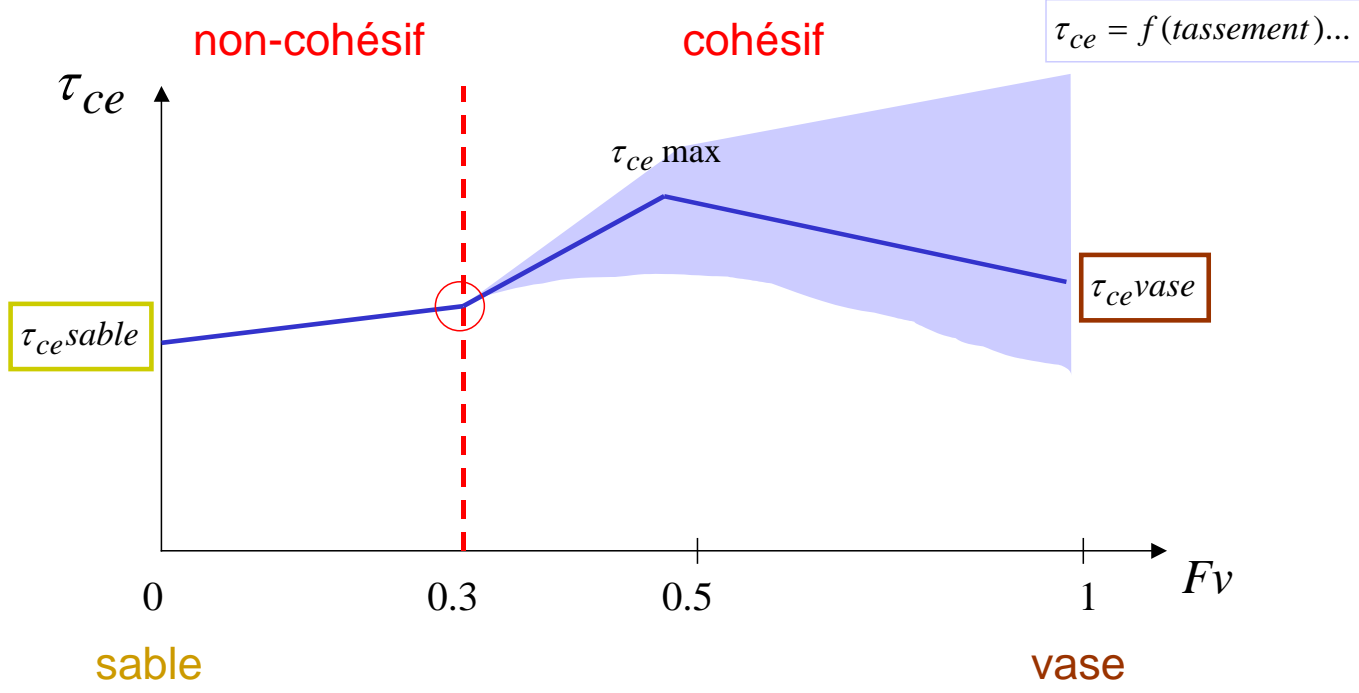

figure 3: variations de la contrainte critique d'érosion en fonction de la fraction massique de vase $F_{v}$

\section{Gestion des fractions de vase et de sable dans le sédiment}

Les fractions respectives de vase et de particules fines dans la colonne de sédiment peuvent varier à chaque pas de temps de transport sédimentaire, en particulier dans la couche superficielle au gré des érosions et des dépôts. Dans le modèle de sédiment multicouche des codes SiAM (e.g. Le Hir et al., 2001b), il a donc été nécessaire de réajuster les épaisseurs de couches et leurs assemblages éventuels, selon que le matériau contenu dans une couche est globalement de type cohésif ou non (paramétré par la fraction massique de vase). En effet, lorsque la couche est de type "sableuse" ( $\left.F v<F v_{c r}\right)$, on considère la fraction volumique de sable figée, les particules fines venant remplir plus ou moins les "vides" restant entre les grains, tandis que dans le cas d'une couche vaseuse, un tassement est possible.

\section{Application à l'embouchure de la Seine, avec le modèle SiAM-3D}

Pour illustrer les possibilités d'une modélisation des transports simultanés de sable et de vase, nous avons choisi le cas de l'embouchure de la Seine, caractérisée par une alternance de fonds de sables fins et de vases (e.g. Lesueur, 1999) et pour laquelle nous disposons d'un modèle hydrosédimentaire tridimensionnel déjà implanté (Le Hir et al., 2001b). Ce modèle résout en différences finies les équations de Navier-Stokes sous approximation hydrostatique et hypothèse de Boussinesq.

Dans la simulation présentée (fig.4: résultats (a) après 3 mois, (b) après 4 mois), deux variables particulaires ont été considérées, les sables fins de diamètre $200 \mu \mathrm{m}$, caractérisés par une vitesse de chute de $2,45 \mathrm{~cm} . \mathrm{s}^{-1}$ et les vases, dont la vitesse de chute varie entre 0,5 et 1,5 $\mathrm{mm} . \mathrm{s}^{-1}$ selon les conditions de floculation. Le transport de vase est pleinement tridimensionnel, tandis que l'advection-diffusion de sable est intégrée selon la verticale (par économie de temps-machine), le terme de dépôt étant calculé avec une concentration extrapolée selon un profil de Rouse. La formulation de l'érosion et la gestion multicouche du sédiment (sans tassement dans cette application) respectent la distinction entre les types de sédiment (cohésif ou non) décrite précédemment, la répartition sable/vase pouvant évoluer dans l'espace et dans le temps. La répartition initiale (arbitraire) de sédiment est telle que l'ensemble de l'estuaire est constitué de sable; un stock de vase lui est superposé dans le chenal de navigation. 
La figure 4 montre que les concentrations de sable et de vase ne se produisent pas aux mêmes endroits : pour les sables, les secteurs de courant de marée maximum (dans le chenal) sont privilégiés, et restent les mêmes quel que soit le débit de la Seine; les vases en suspension, quant à elles, reproduisent la structure du bouchon vaseux, et remontent vers l'amont en étiage. L'examen de l'évolution des fractions dans le sédiment superficiel (figure non présentée) montre que les vases se déposent préférentiellement sur la vasière nord et dans le port du Havre, là où les contraintes de cisaillement sur le fond sont les plus faibles; au contraire, les sables se concentrent à l'"engainement" du chenal là où les gradients des transports horizontaux sont les plus marqués, en conformité avec les observations.

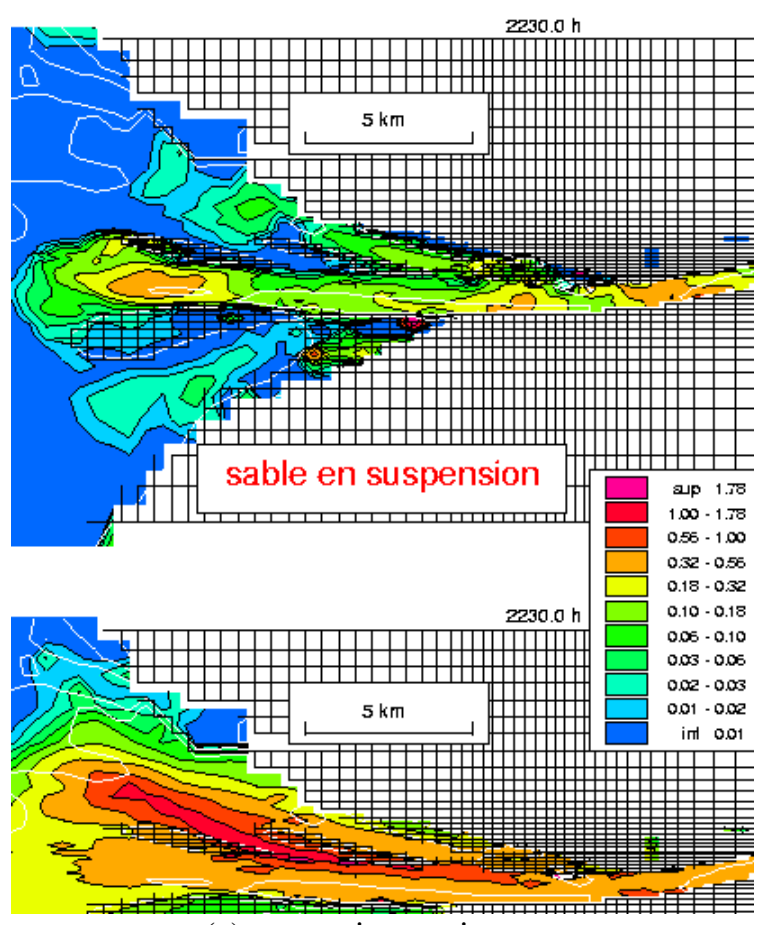

(a)crue - ni vent ni vague

valeur moyenne en vive eau run ressa b18

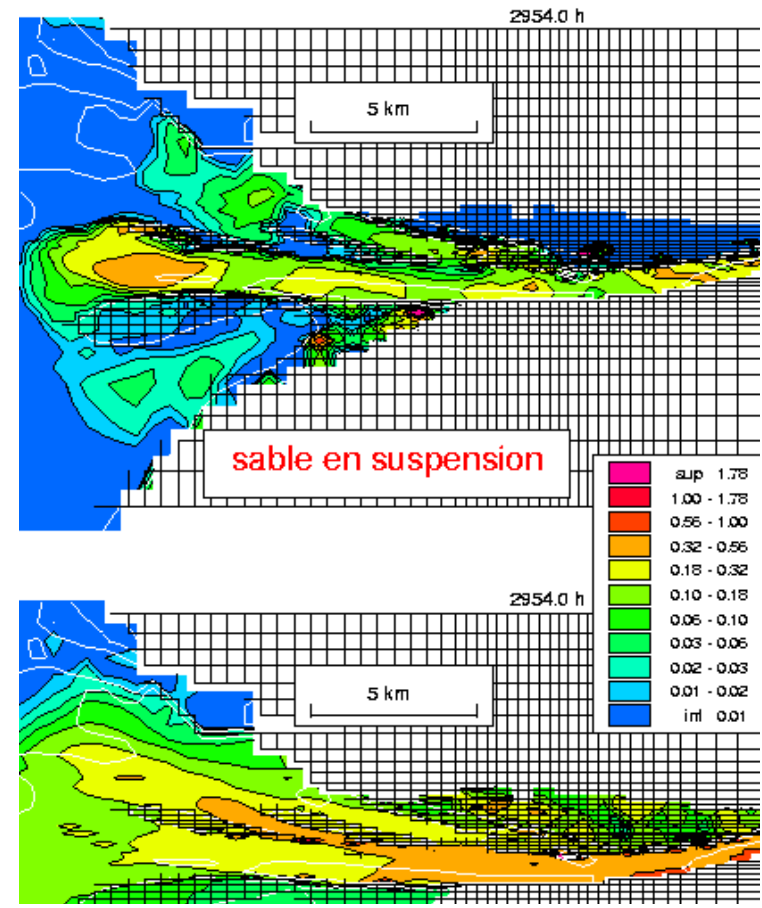

(b)étiage, 1 mois après la crue - ni vent ni vague valeur moyenne en vive eau run ressa b18

Figure 4 : simulation des transports simultanés de sable et de vase à l'embouchure de la Seine (zoom du modèle SiAM-3D de l'estuaire de la Seine) : concentrations moyennes (sur une période de marée) (en $\mathrm{kg} \cdot \mathrm{m}^{-3}$ ) calculées en vive eau .

- (a) : en crue - (b) : 1 mois plus tard, en étiage

\section{Conclusion}

Dans la première partie, nous avons montré qu'un modèle d'advection-diffusion de sable, assorti d'une loi d'érosion adaptée, discrétisé ou non sur la verticale, reproduit les capacités de transport à l'équilibre données dans la littérature, à condition d'extrapoler convenablement (e.g. selon un profil de Rouse) la concentration près du fond dans le terme de dépôt. La sensibilité du résultat à la hauteur de référence choisie (là où le terme de dépôt est évalué) reste à préciser.

Ensuite une adaptation de la formulation de l'érosion a été proposée, pour tenir compte du comportement plus ou moins cohésif des mélanges sable/vase. Cette adaptation, qui entraîne une modification de la gestion des couches sédimentaires, a été introduite dans le modèle SiAM-3D. L'application à l'embouchure de la Seine fournit des résultats encourageants, mais la multiplicité des combinaisons possibles de sable et de vase nécessite des efforts expérimentaux accrus. 


\section{Remerciements}

Ce travail a été réalisé dans le cadre du Programme Scientifique Seine-Aval II, piloté par le Conseil Régional de Haute-Normandie. En particulier ce programme finance la bourse de thèse de Benoît Waeles.

\section{Références}

1.Holly F.M. et Rahuel J.L., 1990. New numerical/physical framework for mobile-bed modelling. Part 1: numerical and physical principles. Journal of Hydraulic Research, $28 \mathrm{~N}^{\circ} 4$, 401-416.

2.Beach, R.A., Sternberg, R.W., 1988. Suspended sediment transport in the surf zone: response to cross-shore infragravity motion. Mar. Geol. 80, 671-679.

3.Chapalain, G. et Thais, L., 2000. Tide, turbulence and suspended sediment modelling in the eastern English Channel. Coastal Engineering, 41 : 295-316.

4.Chesher, T.J. et Ockenden, M.C., 1997. Numerical modelling of mud and sand mixtures. Cohesive sediments (N. Burt, R. Parker and J. Watts editors), pp 395-406.

5.Davies, A.G. et Li, Z., 1997. Modelling sediment transport beneath regular symmetrical and asymmetrical waves above a plane bed. Cont. Shelf Res., 17(5): 555-582.

6.Fredsoe J., O.H. Anderson and S.Silberg, 1986. Distribution of suspended sediment in large waves. Jour. of waterway, Port, Coastal and Ocean Eng., ASCE, 111(6), 1041-1059.

7.Le Hir P., Bassoullet Ph. et Jestin H., 2001a. Application of the continuous modeling concept to simulate high concentration suspended sediment in a macrotidal estuary. In W.H. McAnally and A.J. Mehta (eds) Coastal and Estuarine Fine Sediments Processes, Elsevier, proceedings in Marine Science No 3, 229-247.

8.Le Hir P., Ficht A., Silva Jacinto R., Lesueur P., Dupont J.P., Lafite R., Brenon I., Thouvenin B., Cugier Ph., 2001b.Fine sediment transport and accumulations at the mouth of the Seine estuary (France). Estuaries, Vol. 24, No 6b, 950-963.

9.Lesueur P., 1999. Sables, chenaux, vasières: dynamique des sédiments et évolution morphologique. Fascicule No. 3 du Programme scientifique Seine-Aval., Ed. Ifremer, 39p.

10.Mitchener, H. et Torfs, H., 1996. Erosion of mud/sand mixtures. Coastal Engineering, $29: 1-25$.

11.Panagiotopoulos, I., Voulgaris, G. and Collins, M.B. 1997. The influence of clay on the threshold of movement of fine sandy beds. Coastal Engineering 32: 19-43.

12.Smith, J.D. et McLean, S.R., 1977. Spatially averaged flow over a wavy surface. J. Geophys. Res., 82: 1735-1746.

13.Soulsby, R.L., 1997. Dynamics of marine sands. R. Soulsby and Thomas Telford Services Limited, ISBN:07277 2584 X, 249p.

14.Torfs, H., H. Williamson, H. Huysentruyt et E. Toorman, 1996. Settling and consolidation of mud/sand mixtures. Coastal Engineering, 29:27-45.

15.Van Ledden M., 2003. Sand-mud segregation in estuaries and tidal basins. Ph.D Thesis, Delft University of Technology.

16.Van Ledden M., van Kesteren W., Winterwerp J.C., 2004. A conceptual framework for the erosion behaviour of sand-mud mixtures. Continental Shelf Research 24, 1-11.

17.Van Rijn, L.C., 1984. Sediment pick-up functions. Journal of Hydraulic Engineering, 110(10), 1494-1502.

18.Van Rijn L., 1989. Handbook Sediment Transport by Current and Waves, Report H461, Delft Hydraulics. 